\title{
Fungus- (Alternaria sp.) Mediated Silver Nanoparticles Synthesis, Characterization, and Screening of Antifungal Activity against Some Phytopathogens
}

\author{
Theint Theint Win $\mathbb{D}^{1},{ }^{1,2}$ Sikandar Khan ${ }^{(D)},{ }^{3}$ and PengCheng Fu ${ }^{1}{ }^{1}$ \\ ${ }^{1}$ State Key Laboratory of Marine Resource Utilization in South China Sea, Hainan University, 58 Renmin Avenue, Haikou, \\ Hainan 570228, China \\ ${ }^{2}$ Biotechnology Research Department, Ministry of Education, Kyaukse 05151, Myanmar \\ ${ }^{3}$ Department of Biotechnology, Shaheed Benazir Bhutto University, Sheringal, KP 18000, Pakistan
}

Correspondence should be addressed to PengCheng Fu; pcfu@hainanu.edu.cn

Received 28 September 2020; Revised 7 November 2020; Accepted 16 November 2020; Published 29 November 2020

Academic Editor: Marco Rossi

Copyright (c) 2020 Theint Theint Win et al. This is an open access article distributed under the Creative Commons Attribution License, which permits unrestricted use, distribution, and reproduction in any medium, provided the original work is properly cited.

\begin{abstract}
The scientific consensus is now on developing a biocontrol agent that can cause cellular metabolic reprogramming against agricultural pathogens. Biosynthesis of silver nanoparticles was performed by using phytopathogenic fungi (Alternaria sp.) isolated from banana cultivated soil. Alternaria sp. can grow very fast and produce high enough bioactive compounds. This study aims to biosynthesize silver nanoparticles (AgNPs) using fungal Alternaria sp.'s metabolites as a safe antifungal agent against plant pathogenic fungi (Fusarium spp. and Alternaria sp.). To visualize the formation of AgNPs, analytical instruments were used, such as ultraviolet-visible (UV-Vis) spectroscopy, Fourier-transform infrared (FTIR) spectroscopy, scanning transmission electron microscopy (STEM), energy dispersive X-ray (EDX), and elemental mapping. The UV-visible spectra showed a peak at $435 \mathrm{~nm}$. Analysis of scanning transmission electron microscopy (STEM) micrographs evidenced that the size of synthesized silver nanoparticles ranged between 3 and $10 \mathrm{~nm}$. The resulting AgNPs showed distinct antifungal activity against selected plant pathogenic fungi. Synthesized AgNPs have demonstrated remarkable potential for the use of antifungal compounds to combat plant diseases.
\end{abstract}

\section{Introduction}

Biological control agents should be prepared with less risk to human and livestock [1]. Currently, the use of biological control agents at the commercial level has some limitations, such as adaptability to biotic and abiotic factors and deterioration of the desired activity between in vitro and in vivo [2]. It is time to develop more effective and nonpersistent biopesticides with the aid of nanotechnology using biogenic silver, zinc, copper, gold, and iron [3].

Fungitoxic metabolites (antibiotics and other bioactive metabolites) are shown to contribute to biocontrol applications in agriculture [4]. Fungi can produce a considerable amount of extracellular enzymes such as chitinases, glucanases, proteases, glycosyl hydrolases, xylanases, cellulases, and mannanases under suitable conditions [5]. Because of their conformity with the ideals and concepts of green chemistry, metallic nanoparticles from biological sources have received a great deal of attention [6].

Nanosized silver particles have become more popular as technological advancements make their processing more economical [7]. Many attempts have been made to synthesize nano-based antifungal compounds from zinc oxide and silver nitrate used for the effective management of plant pathogenic fungus, including Fusarium oxysporum, Penicillium digitatum, Alternaria citri, Alternaria alternate, and Aspergillus niger $[8,9]$. Copper nanoparticles that exhibited strong antifungal activities against tested plant pathogenic fungi responsible for crop diseases were also synthesized by Kanhed et al. (2014) and Bramhanwade et al. (2016) [10, 11]. 
One of the possible ways in which silver can be used is the control of plant diseases. Since silver displays several modes of inhibitory action on microorganisms [12], it can be used relatively safer than synthetic fungicides [13]. The efficacy and wide range of antimicrobial activity of AgNPs against plant phytopathogenic fungi were also shown by Al-Zubaidi et al. (2019) [14]. However, the restricted study has so far demonstrated its applicability of silver for managing plant diseases [13].

For the control of some plant fungal diseases, biogenic nanoparticles have proved to be effective nanomaterialbased fungicides. Therefore, they possess the potential for extensive use in agriculture as biocontrol agents for promoting sustainable agriculture [3]. This study aimed to synthesize environmentally-friendly biogenic AgNPs by using metabolites from a fast-growing fungus Alternaria sp. for the effective control of some phytopathogenic fungi. Alternaria sp.'s extracts were used for the first time in this study and were found to be highly effective in the reduction of silver ions into silver nanoparticles. Synthesis of metal NPs based on fungal extract is found to be the most effective and environmentally-friendly way of preparing biocontrol agents for phytopathogens suppression.

\section{Materials and Methods}

2.1. Reagents and Instruments. Silver salts (analytical grade) and culture media were purchased from Aladdin, China, and the solvent used throughout the experiments was double distilled water $\left(\mathrm{ddH}_{2} \mathrm{O}\right)$ (Millipore $18.2 \mathrm{MX} \mathrm{cm}$ ). Instruments used in this study were high-speed refrigerated centrifuge (JW-3021HR), UV/Vis spectrophotometer (Shimadzu, UV-3600), scanning transmission electron microscope (STEM), energy-dispersive X-ray (EDX) (JEOL JEMARM200F $200 \mathrm{kV}$ ), zeta potential (HORIBA Zetasizer SZ100), and FTIR (Nicolet 6700 FTIR spectrometer).

\subsection{Fungal Isolation, Characterization, and Preparation of} Metabolites. Alternaria sp. was isolated from severely wilt affected banana plants rhizosphere soil in the way below: soil samples were collected from Guilin, China. The serial dilution method was used to isolate fungal pathogens and cultured on PDA (potato dextrose agar) plates, which were incubated at $26-28^{\circ} \mathrm{C}$ for 5 days. Purified cultures were visually identified using their cultural and microscopic morphology. Presumptive identifications were confirmed with internal transcribed spacer (ITS) rDNA sequence analysis. The entire ITS region of the fungal isolates was amplified with the primer pair ITS1F ( $5^{\prime}$-CTTGGTCATTTAGAGGAAGTAA-3') and ITS4 $\left(5^{\prime}\right.$-TCCTCCGCTTATTGATATGC-3') [15]. The resulting amplicons were sequenced by a $3730 \mathrm{XL}$ DNA analyser (Applied Biosystems, USA). Sequence identification was performed and deposited to NCBI, BLAST database (https://blast.ncbi.nlm.nih.gov/Blast.cgi?

PAGE=Nucleotides). Its accession number is MN096578.

Alternaria sp. (seven days old) was grown in $200 \mathrm{ml}$ of $\mathrm{PDB}$ (potato dextrose broth) at $26-28^{\circ} \mathrm{C}$ for 5 days. Biomass was recovered by centrifugation at $7000 \mathrm{rpm}$ for $10 \mathrm{~min}$. The supernatant was decanted, and the biomass pellet was subsequently washed with sterilized deionized water to remove all the remaining components of the growth medium. After washing, the biomass was resuspended in $100 \mathrm{ml}$ of sterilized $\mathrm{ddH}_{2} \mathrm{O}$, incubated at $28^{\circ} \mathrm{C}$ for 3 days, and then filtered through a $0.22 \mu \mathrm{m}$ membrane filter to get smallmolecular-weight metabolites. The resulting filtrates were used for silver nanoparticles synthesis [16].

2.3. Extracellular Biosynthesis of Silver Nanoparticles. For the preparation of AgNPs, $10 \mathrm{ml}$ of the Alternaria sp.'s metabolic filtrate was treated with $50 \mathrm{ml}$ of $1 \mathrm{mM}$ silver nitrate $\left(\mathrm{AgNO}_{3}\right)$ solution and kept on stirring $(500 \mathrm{rpm})$ at room temperature $\left(25 \pm 1^{\circ} \mathrm{C}\right)$ [16]. The development of reddish brown color in the reaction mixture designates the synthesis of AgNPs. Optical property such as localized surface plasmon resonance (LSPR) for the AgNPs was identified by UVvisible spectrometry (Shimadzu UV3600, Japan). The reaction was terminated after SPR bands saturation. The acquired product in suspension was recovered by centrifugation at $12,000 \mathrm{rpm}$ for $15 \mathrm{~min}$. The collected AgNPs were repeatedly washed and freeze-dried. The fungimediated green synthesis of silver nanoparticles was further used for characterizing their properties and examining their antifungal activity against phytopathogens [17].

\subsection{Characterization of Silver Nanoparticles}

2.4.1. UV-Visible Spectroscopy Analysis. AgNPs detection was primarily performed by visual examination of fungal filtrate color change after silver nitrate treatment. The appearance of dark brown fungal cell filtrate suggests AgNPs formation. Besides, the biogenic AgNPs synthesis was further confirmed using UV-Vis absorption spectra. Optical property such as localized surface plasmon resonance (LSPR) for the AgNPs was identified by UV-visible spectrometry (Shimadzu UV3600, Japan). The reaction was terminated after SPR band saturation. The absorption spectra of the samples were measured using a spectrophotometer (Shimadzu UV-3600, Japan) in the wavelength range of $190-800 \mathrm{~nm}$; $\mathrm{ddH}_{2} \mathrm{O}$ distilled water was used as blank.

2.4.2. Scanning Transmission Electron Microscope (STEM) and Energy-Dispersive X-Ray (EDX) Analysis. To prepare dried AgNPs powder in the fungal metabolites, treated broth was centrifuged at $12000 \mathrm{rpm}$ for 15 minutes. Supernatants were discarded, and AgNPs pellets were washed three times with autoclaved distilled water and freeze-dried [16]. The dried AgNPs were dispersed and transferred to $\mathrm{Cu} 200$ mesh TEM grids having few $\mathrm{nm}$ thick carbon film and dried for $24 \mathrm{~h}$ at $60^{\circ} \mathrm{C}$. The AgNPs were analyzed using a JEOL JEMARM200F $200 \mathrm{kV}$ STEM. The instrument was equipped with a light element $100 \mathrm{~mm}^{2}$ SDD EDS detector. Mineralogical information and two-dimensional elemental maps were obtained for $\mathrm{Ag}, \mathrm{C}$, and $\mathrm{K}$ by electron diffraction of selected areas using a spatial resolution of $50 \mathrm{~nm}$. The particle size 
was estimated by the SEM result and confirmed by the STEM result.

2.4.3. Zeta Potential. In order to determine the surface electric charge of dried AgNPs as prepared above, zeta potential measurement was carried out by using Zetasizer (Zetasizer SZ-100, HORIBA) [18].

2.4.4. Fourier-Transform Infrared (FTIR). The freeze-dried powder of AgNPs as prepared above was subjected to FTIR analysis. FTIR spectral bands in the prepared materials (AgNPs) were determined using the FTIR spectrometer (Nicolet 6700, Thermo Scientific), $400-4000 \mathrm{~cm}^{-1}$ in transmittance mode. Samples for FTIR analysis were prepared using the potassium bromide $(\mathrm{KBr})$ pellet technique, which involves mixing the AgNPs thoroughly with $\mathrm{KBr}$ before forming a pellet at high pressure.

2.5. Phytopathogens Collection. The plant-pathogenic fungi used in this study were Alternaria sp., Fusarium oxysporum, Fusarium moniliforme, and Fusarium tricinctum. Alternaria sp. was isolated from banana rhizospheric soil. The latter three were provided by Dr. Jing LV, State Key Laboratory of Heavy Oil Processing, University of Petroleum, Beijing, China. All the fungi were grown on potato dextrose agar (PDA) according to the manufacturer's instructions. Fusarium oxysporum appeared as an abundant cottony white colony with white aerial mycelium. Fusarium moniliforme had a black colony on the backside with a white aerial and marginal mycelium. Fusarium tricinctum colony had abundant cottony white mycelium.

\subsection{Screening of Antifungal Potential of Silver Nanoparticles.} The well diffusion method was used to assess the antifungal potential of the biosynthesized AgNPs against four pathogenic strains (Fusarium oxysporum, Fusarium moniliforme, Fusarium tricinctum, and Alternaria sp.). Petri dishes were prepared with PDA agar medium having wells of $8 \mathrm{~mm}$ diameter at equal distance. After inoculation, the wells were loaded with different concentrations $(25,50$, and $100 \mu \mathrm{l})$ from the stock solution of AgNPs $\left(1 \mathrm{mg} \cdot \mathrm{ml}^{-1}\right)$. The plates were incubated at $28 \pm 1^{\circ} \mathrm{C}$, and after 5 days the inhibition zones were examined [19]. Comparison of the means of inhibition of mycelial growth was subjected to one-way ANOVA in order to identify the significance of the activity. The significant differences in each activity were determined by the $t$-test $(p=0.05)$.

\section{Results and Discussion}

3.1. Synthesis of Nanoparticles and UV-Vis Spectral Analysis. Alternaria sp. was isolated and grown on plates for extract preparation (Figure 1(a)). The microscopic morphology of the fungal spore is shown in Figure 1(b). Phylogenetic tree of Alternaria sp. and related species based on confidently ITS sequences constructed with neighbor-joining is presented in Figure 1(c). After mixing the Alternaria sp. extracts with the
$\mathrm{AgNO}_{3}$ solution, it was noted visually that the color of the mixture was changed from transparent to dark brownish (Figure 2). The color changed to dark brown as a consequence of the reduction of silver ions with the formation of AgNPs [18]. After $30 \mathrm{~min}$ of reaction, both alterations in color and the absorbance were recorded at regular intervals, as the color change was the first evidence of success in nanoparticle formation. Generally, the color change occurred due to reducing agents released from fungal extracts into the solution [16]. This was also supported by UV-visible spectra; when exposed to the light of a specific wavelength, the NPs displayed a unique surface phenomenon called surface plasmon resonance (SPR); due to this, a specific peak formation occurred for each kind of NPs by UV-Vis spectroscopy [20]. The synthesis of AgNPs using (Alternaria sp.) extract was monitored using UV-Vis spectroscopy; the light absorption pattern of the fungal biomass was observed in the range of $350-500 \mathrm{~nm}$.

The silver ions reduction peak of the SPR occurred at $435 \mathrm{~nm}$ (Figure 3). The same peak area for AgNPs at $435 \mathrm{~nm}$ was also reported by other researchers; one of them used incubation of $\mathrm{Ag}$ ions with fungal biomass of Trichoderma koningii [19]. Spectral analysis showed that the UV-Vis SPR peak at $435 \mathrm{~nm}$ typically observed for AgNPs that without altering the peak position increased as a function of time. It further confirmed the conversion of silver ions into silver nanoparticles. This observation suggested the Alternaria sp. mediated the biogenic formation of monodispersed AgNPs. It is obvious that SPR peaks of metal nanoparticles depend on particle size, shapes, and reaction medium. The SPR peaks normally show a redshift with an increase in the particle size [21]. In this study, the SPR peak retained its spectral position, confirming a uniform size particle distribution. Fungal extracts are rich in bioactive molecules and functional groups like - $\mathrm{OH}$, amide, and carboxylic groups, which improved the quantitative production of AgNPs and their stable dispersion [22]. Therefore, fungal extracts have the power to be used for the biogenic synthesis of nanoparticles.

We measured UV-Vis spectra of the reacting sample in regular time intervals of $30 \mathrm{~min}$ to $24 \mathrm{hr}$ to study the effect of the reaction time. Initially, the color of the filtrate was paleyellow. As time passes, the color shifts to brown, followed by dark brown with increased absorbency, which suggests the continuous synthesis of AgNPs in filtrate (Figure 3). Moreover, the stability was evaluated after 30 days of synthesis and there was no change in absorption peak value, indicating that the nanoparticles are highly stable after one month under ambient conditions $\left(28^{\circ} \mathrm{C}\right)$. Only one plasma band is obtained in the case of monodispersed nanoparticles. The increased intensity indicates a progressive degree of reaction with a corresponding increase in the number of particles [16].

\subsection{Characterization of Fungi-Based Synthesis of AgNPs}

3.2.1. Zeta Potential Analysis. The zeta potential analysis of the biosynthesized AgNPs was found as a single sharp peak 


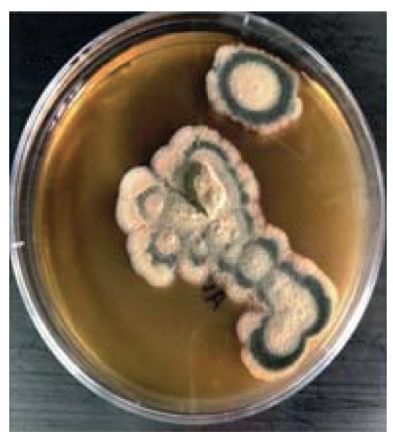

(a)

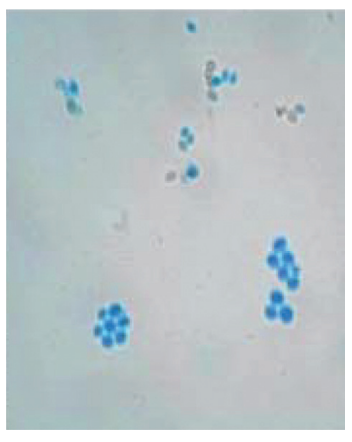

(b)

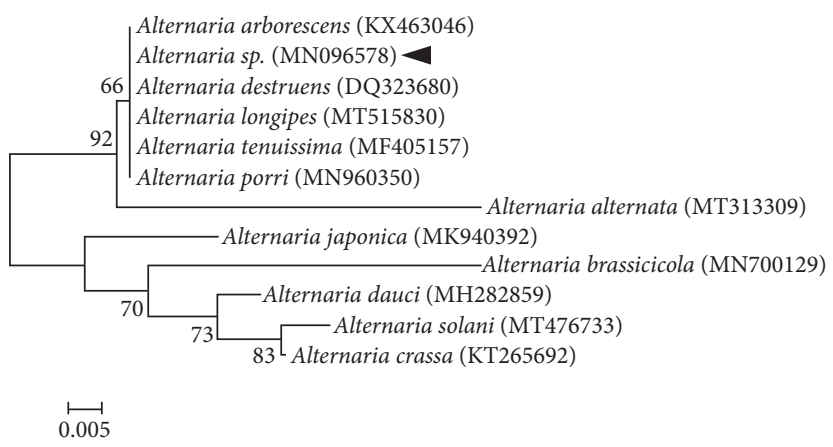

(c)

Figure 1: (a) Macroscopic and Microscopic morphology of Alternaria sp. (b) Phylogenetic tree of Alternaria sp. and related species based on confidently ITS sequences constructed with neighbor-joining implemented in MEGA 5.0. (c) Strain (MN096578) indicates isolate of this study.

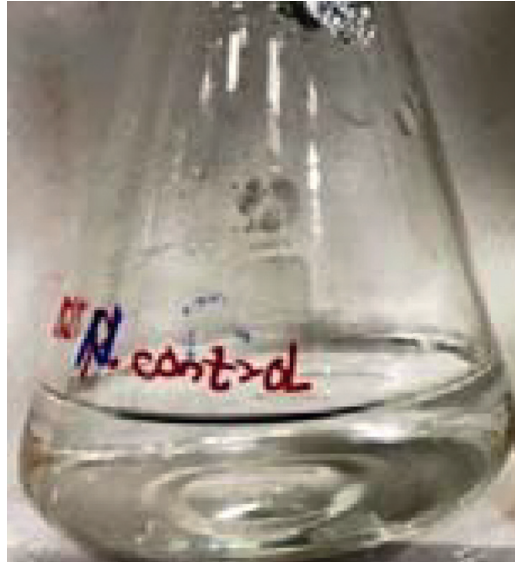

(a)

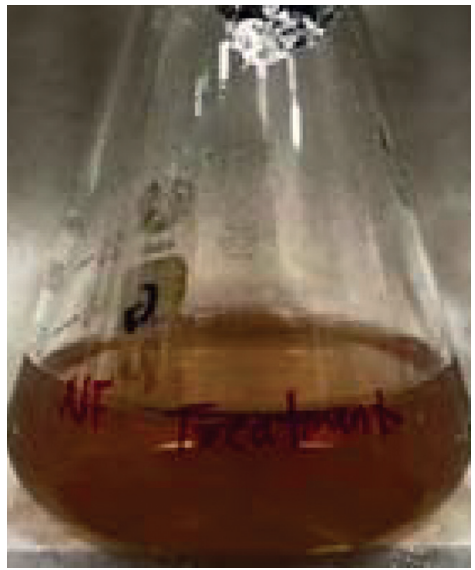

(b)

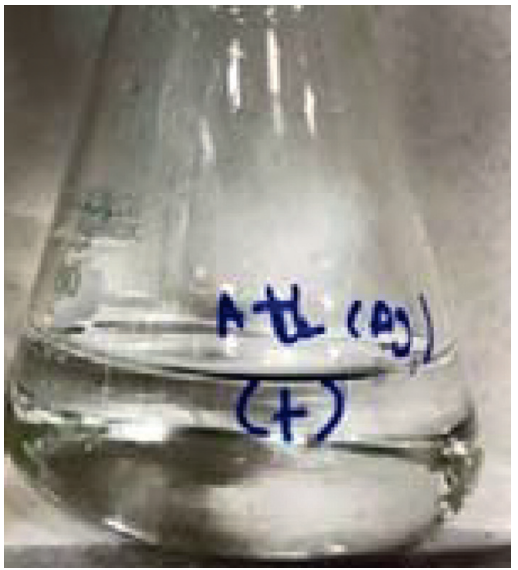

(c)

Figure 2: Color changes after AgNPs synthesis. (a) Fungal metabolites only. (b) Fungal metabolites $+1 \mathrm{mM} \mathrm{AgNO}_{3} 1: 5 \mathrm{v} / \mathrm{v}$. (c) ddH $\mathrm{m}_{2} \mathrm{O}$ $+1 \mathrm{mM} \mathrm{AgNO}_{3}$ 1:5 v/v.

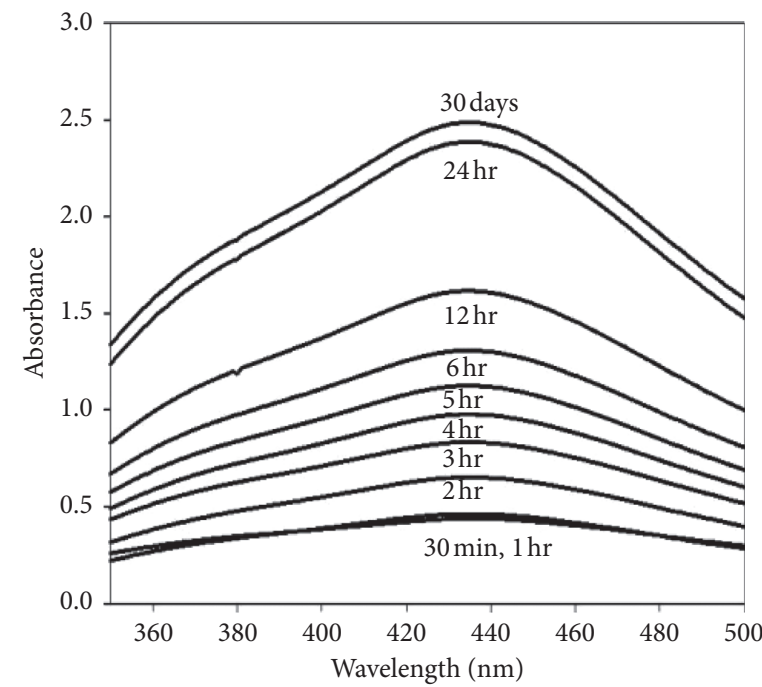

FIGURE 3: SPR banding patterns of AgNPs $(435 \mathrm{~nm}$ ) recorded with UV-Vis spectrometry at regular time intervals of 30 min up to 30 days. 
between -60 and $0 \mathrm{mV}$ while having a maximum intensity at $-31.9 \mathrm{mV}$ (Figure 4). It indicates that the negatively charged moieties are present on the surface of the AgNPs that expanded in the medium. The repulsion among the particles might be due to the negative values that proved that the particles are very stable. Low zeta potential values of particles suggest no flocculation and no tendency to assemble due to repulsion forces among particles [23, 24].

\subsubsection{Scanning Transmission Electron Microscopic (STEM)} Analysis. Morphology and size distribution of the biosynthesized AgNPs were revealed via STEM analysis. The prepared AgNPs exhibited a spherical morphology (Figures 5(a) and 5(b)). The biogenic AgNPs displayed a particle size range of $3-10 \mathrm{~nm}$ (Figure $5(\mathrm{c})$ ). It is also evident that the biomolecules in the extracts of Alternaria sp. promoted the synthesis of AgNPs. The SEM images of AgNPs reported by other researchers from different extracts showed spherical particles, aggregated spherical particles, irregularly shaped particles, and cubic particles $[16,25]$. The moderate particles' sizes observed were 5, 12, 25, 35, and $50 \mathrm{~nm}$, for AgNPs synthesized by different biological extracts using water as a solvent. Similar results were also found by other researchers recently $[16,25-28]$. In an article comparing the advantages and drawbacks of these methods and the applications of nanoparticles in various domains, a synthesis of chemical, physical, and biological methods for obtaining AgNPs of different shapes and dimensions (from 2 to $300 \mathrm{~nm}$ ) was described [29].

3.2.3. Energy-Dispersive X-Ray (EDX) Analysis. EDX of the AgNPs was performed to investigate the elemental composition of the biosynthesized AgNPs (Figure 5(d)). The EDX spectra revealed the presence of silver peaks around 3 and $3.1 \mathrm{keV}$, which appeared due to the discharge of different electrons from $\mathrm{L}$ and $\mathrm{K}$ shells of silver, respectively. Therefore, the EDX pattern clearly indicates that the AgNPs are crystalline in nature. The lower energy peak $(3 \mathrm{keV})$ was responsible for the outer shell electrons (L), and a higher energy peak $(3.1 \mathrm{keV})$ was responsible for inner shell electrons $(\mathrm{K})$. The observation was in agreement with the previous report by Kasithevar et al. [27]. The carbon peak present in the spectra was mainly due to the carbon adhesive tape used, and the rest of the peaks might be due to inorganic impurities in the biomolecules from the Alternaria sp. extract. The EDX data of AgNPs showed that the weight percentage of $\mathrm{Ag}$ was $92 \%$. This is also in agreement with the results reported earlier [28].

3.2.4. FTIR Spectral Analysis. FTIR was employed to quantify and determine the functional biomolecules in the Alternaria sp. extracts, which were important for reducing silver ions into relative silver nanoparticles. The Alternaria sp. extracts have a variety of biomolecules, which might be involved in the synthesis of these AgNPs (Figure 6). The occurrence of many prominent peaks in the IR region of the electromagnetic spectrum is due to different functional

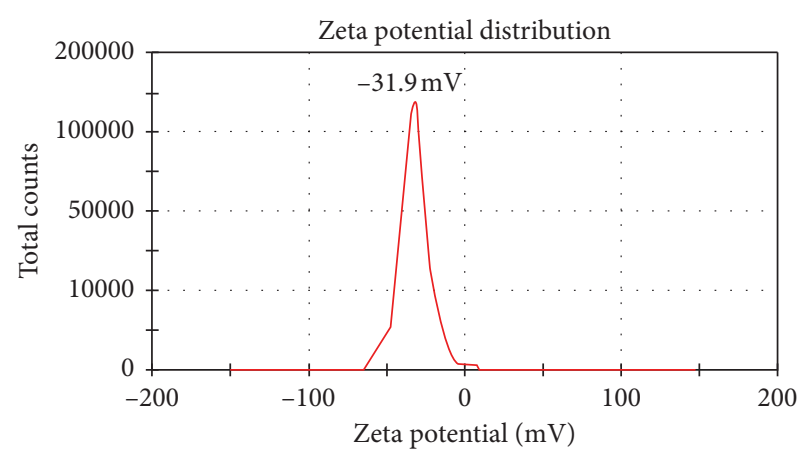

Figure 4: Zeta potential of biosynthesized silver nanoparticles illustrating the surface charge value.

groups. The active and extended band was observed at $3421 \mathrm{~cm}^{-1}$ that confirmed the presence of polyphenolic $-\mathrm{OH}$ group. The second band was observed relative to the alkyl group of $\mathrm{C}-\mathrm{H}$ stretching vibrations at the absorption band $2870 \mathrm{~cm}^{-1}$; the third narrowband occurred at $2815 \mathrm{~cm}^{-1}$ is ascribed to $\mathrm{C}-\mathrm{H}$ of alkane group. The fourth peak at $1638 \mathrm{~cm}^{-1}$ indicating the presence of amide I group; the fifth short band appeared at $1450 \mathrm{~cm}^{-1}$ assigned to carboxylic acid while the sixth peak around $1156 \mathrm{~cm}^{-1}$ is representing stretching of the aromatic ring, and the seventh band at $1027 \mathrm{~cm}^{-1}$ corresponds to $\mathrm{C}-\mathrm{N}$ stretching vibrations of the aliphatic amines of protein. The last wide and short band at $470 \mathrm{~cm}^{-1}$ represented alkyl halides. This investigation also demonstrated that protein and amino acids are the main components and have the capacity to bind metals. It is evident that polysaccharides, sulfonated compounds, and amide linkages are strongly involved in the reduction of silver ions into nanoparticles. The biomolecules present in fungal extract have dual functions of reducing silver ions and stabilizing the NPs $[26,30]$.

3.3. Antifungal Potential of the AgNPs. The well diffusion method was used to assess the antifungal potential of the biosynthesized AgNPs against four phytopathogenic strains of fungi (Fusarium oxysporum, F. maniliforme, F. tricinctum, and Alternaria sp.). Recent research of the antifungal activity of AgNPs was obtained by green synthesis mediated by different plants and microbes [19, 31, 32], evaluated primarily by agar well diffusion method. This is consistent with the results of our research and showed comparable values representing antifungal action. Twelve Petri dishes were prepared with PDA medium having wells of $8 \mathrm{~mm}$ diameter at equal distance. After inoculation, the wells were loaded with different concentrations $(25,50$, and $100 \mu \mathrm{l})$ from a stock solution of AgNPs $\left(1 \mathrm{mg} \cdot \mathrm{ml}^{-1}\right)$. The plates were incubated at $28 \pm 1^{\circ} \mathrm{C}$, and after 5 days, the inhibition zones were measured (Figure 7). Table 1 summarizes the inhibition zone diameter of AgNPs against four phytopathogens.

The minimum inhibitory concentration (MIC) of AgNPs was $25 \mu \mathrm{l}$ while the growth rates of all strains of fungi were quite low at 50 and $100 \mu$ l. The diameter of inhibition zones was measured as a score, indicating that the inhibition zones' diameter increased with increasing 


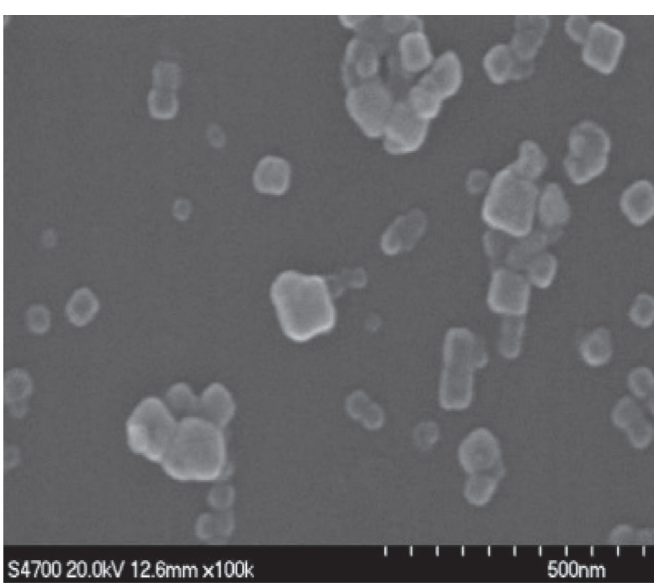

(a)

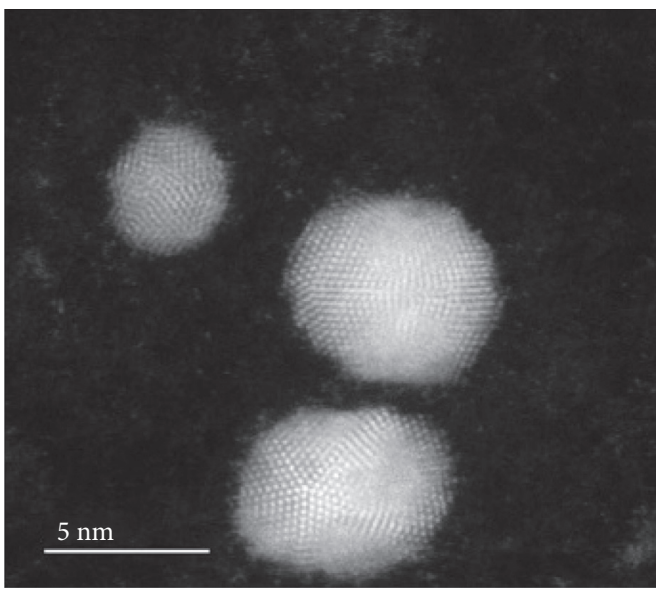

(c)

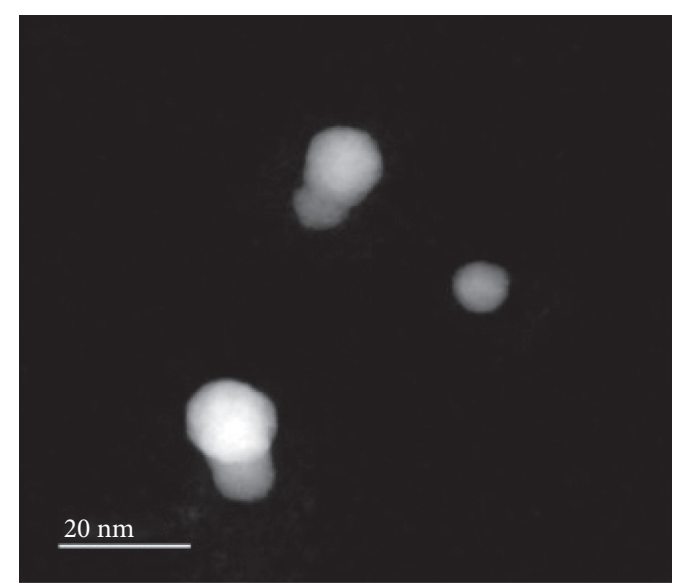

(b)

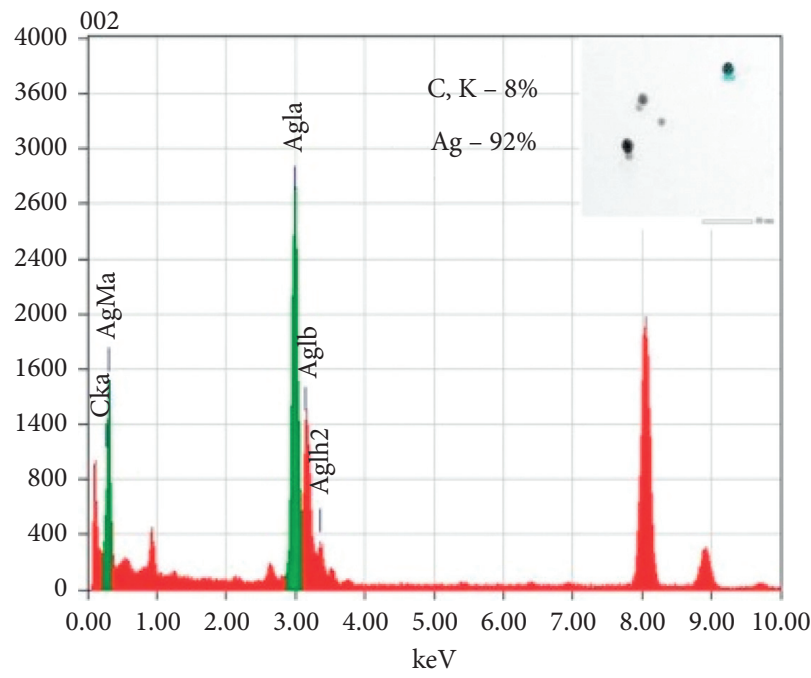

(d)

Figure 5: (a) SEM micrograph of AgNPs synthesized using Alternaria sp. (scale bar-500 nm), (b) STEM of AgNPs showing dispersed particles (scale bar-20 nm), (c) STEM image of the silver nanoparticle (scale bar $-5 \mathrm{~nm}$ ), and (d) EDX spectrum of AgNPs confirming its composition.

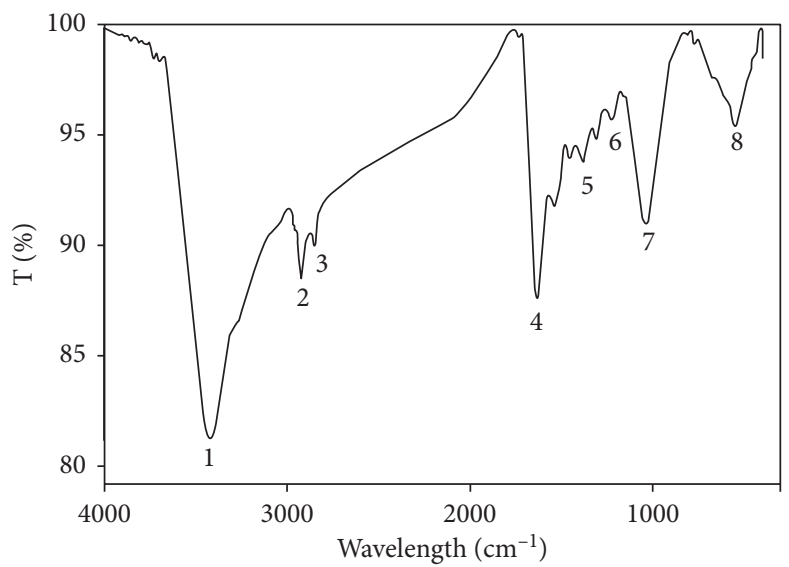

FIGURE 6: FTIR spectra of biosynthesized AgNPs using Alternaria sp. $1=$ phenolic-OH group, $2=\mathrm{C}-\mathrm{H}$ stretching vibrating of alkyl group, $3=\mathrm{C}-\mathrm{H}$ stretching vibrating of alkane group, $4=$ amide group, $5=$ carboxylic acid, $6=\mathrm{C}-\mathrm{C}$ stretching aromatic group, $7=\mathrm{C}-\mathrm{N}$ stretching, and $8=$ alkyl halide. 


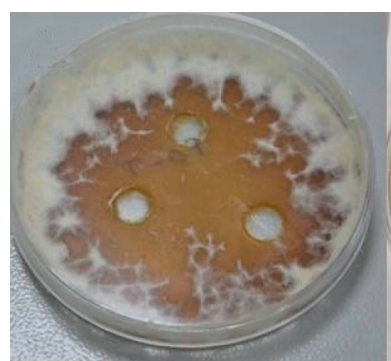

(a)

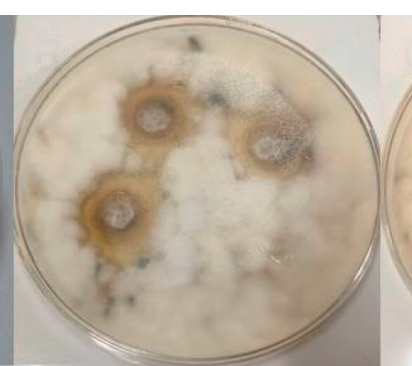

(b)

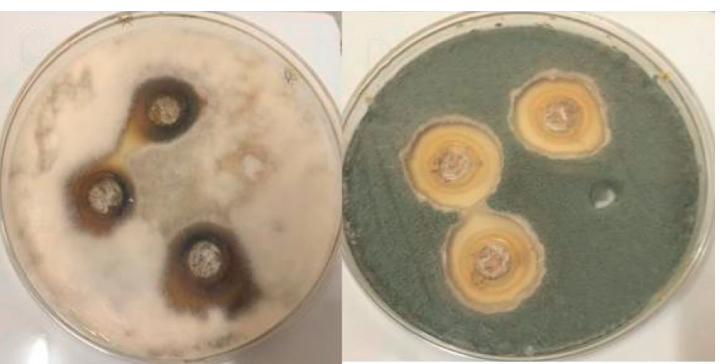

(c) (d)

Figure 7: Antifungal activity of silver nanoparticles $(50 \mu \mathrm{l})$ against (a) Fusarium oxysporum, (b) Fusarium moniliforme, (c) Fusarium tricinctum, and (d) Alternaria sp.

TABle 1: Antifungal activity of silver nanoparticles against four phytopathogens.

\begin{tabular}{lccc}
\hline \multirow{2}{*}{ Phytopathogens } & \multicolumn{3}{c}{ Inhibition zone diameter $(\mathrm{mm})$} \\
& $25 \mu \mathrm{l}$ & $50 \mu \mathrm{l}$ & $100 \mu \mathrm{l}$ \\
\hline Fusarium oxysporum & $14.7 \pm 1.5^{\mathrm{ab}}$ & $17 \pm 2^{\mathrm{a}}$ & $21.3 \pm 1.0^{\mathrm{a}}$ \\
Fusarium moniliforme & $9 \pm 2^{\mathrm{c}}$ & $11.3 \pm 1.5^{\mathrm{b}}$ & $17.5 \pm 1.3^{\mathrm{b}}$ \\
Fusarium tricinctum & $11 \pm 2^{\mathrm{bc}}$ & $11.5 \pm 1.3^{\mathrm{b}}$ & $21.2 \pm 1.0^{\mathrm{a}}$ \\
Alternaria sp. & $17.1 \pm 1.02^{\mathrm{a}}$ & $19.5 \pm 1.5^{\mathrm{a}}$ & $21.6 \pm 1.5^{\mathrm{a}}$ \\
\hline
\end{tabular}

the concentration of AgNPs. The average diameters of inhibition zones were $14.7 \pm 1.5 \mathrm{~mm}, 17 \pm 2 \mathrm{~mm}$, and $21.3 \pm 1 \mathrm{~mm}$ at 25, 50, and $100 \mu \mathrm{l}$, respectively, for Fusarium oxysporum (Figure 7(a)). Inhibition zones for Fusarium moniliforme were measured as $9 \pm 2 \mathrm{~mm}, 11.3 \pm 1.5 \mathrm{~mm}$, and $17.5 \pm 1.3 \mathrm{~mm}$ at 25,50 , and $100 \mu \mathrm{l}$ concentrations of AgNPs, respectively (Figure $7(\mathrm{~b})$ ). Inhibition zones for Fusarium tricinctum were $11 \pm 2 \mathrm{~mm}, 11.5 \pm 1.3 \mathrm{~mm}$, and $21.2 \pm 1 \mathrm{~mm}$ at 25, 50, and $100 \mu \mathrm{l}$ concentrations of AgNPs, respectively (Figure $7(\mathrm{c})$ ). For Alternaria sp., the average diameters of inhibition zones were $17.1 \pm 1.02 \mathrm{~mm}, 19.5 \pm 1.5 \mathrm{~mm}$, and $21.6 \pm 1.5 \mathrm{~mm}$ at 25,50 , and $100 \mu \mathrm{l}$, respectively (Figure $7(\mathrm{~d})$ ). The maximum inhibition zone was observed at $100 \mu \mathrm{l}$ concentration of AgNPs for Alternaria sp. $(21.6 \pm 1.5 \mathrm{~mm})$ following Fusarium oxysporum $(21.3 \pm 1 \mathrm{~mm})$, suggested the efficacy of the biosynthesized NPs against the phytopathogenic fungal strains. Birla et al. (2013) comparatively studied antimicrobial activity of both chemical $\mathrm{AgNO}_{3}$ and biogenic AgNP; higher inhibition zone diameter values were observed for the pathogenic fungi by biogenic AgNPs [16]. It was found that the inhibition zone increased as the concentration of AgNPs increased. This may be due to the high solution density, which could saturate and cohesively deactivate the fungal hypha [32]. It has also assumed that $\mathrm{Ag}^{+}$mainly affects membrane-bound enzyme functions, such as those in the respiratory chain, leading to loss of cellular integrity and osmotic balance resulting in acute cell toxicity [33-35]. The observed antifungal activity in this study may be due to the destruction of membrane integrity. According to the previous studies, this is because AgNPs bind and anchor to the cell surface. This interaction causes structural changes and damage that significantly disrupts essential cell functions such as permeability, causes pit and gaps to depress respiratory chain enzyme activity, and eventually leads to cell death [36-38]. In conclusion, AgNPs exhibited strong antifungal effects on tested fungi, possibly by destroying the membrane's integrity; thus, it was concluded that AgNPs had considerable antifungal activity, which required further investigation for field applications. To conclude, AgNPs have shown strong antifungal effect, possibly by destructing membrane integrity, on fungi tested; thus, AgNPs were assumed to have a significant antifungal activity, requiring further investigation for clinical applications.

\section{Conclusions}

The green reduction and biogenic synthesis of nanoparticles is an effective, low-cost, sustainable, and environmentallyfriendly approach. The phytopathogenic Alternaria sp.'s extract was used for the first time in this study and was found to be highly effective in the reduction of silver ions into silver nanoparticles. Compared with the earlier various biological extracts reported elsewhere, the Alternaria sp. was found to be more effective in silver reduction, as the NPs obtained as a result of treatment with this extract has the lowest zeta potential $(-31.9 \mathrm{mV})$. Furthermore, the fungi-mediated AgNPs were utilized as efficient biocontrol agents for suppressing various fungal phytopathogens. The maximum inhibition zones $21.6 \pm 1.5 \mathrm{~mm}$ and $21.3 \pm 1.0 \mathrm{~mm}$ were observed at $100 \mu \mathrm{l}$ concentration of AgNPs for Fusarium oxysporum and Alternaria sp., respectively. This suggests the efficacy of the biosynthesized NPs against the fungal phytopathogens. It is concluded that fungal extract-based synthesis of metal NPs is the most efficient and environmentally-friendly way to prepare biocontrol agents for phytopathogens suppression. The parameters for synthesis and in vivo antifungal activity should be carefully studied in the future in order to make use of the full potential of AgNPs from Alternaria sp.'s metabolites, which have shown themselves to be a possible antifungal agent to control phytopathogens.

\section{Data Availability}

No data were used to support this study.

\section{Conflicts of Interest}

The authors declare that they have no conflicts on interest presented in this paper. 


\section{Acknowledgments}

TTW and PCF are thankful for the financial support by the Research Start-Up Funds from Hainan University in China (KYQD_ZR2017212). SK is grateful for the support by Shaheed Benazir Bhutto University, Pakistan.

\section{References}

[1] M. Sastry, A. Ahmad, M. I. Khan, and R. Kumar, "Biosynthesis of metal nanoparticles using fungi and actinomycete," Current Science, vol. 85, pp. 162-170, 2003.

[2] T. H. Askary, "Limitations, research needs and future prospects in the biological control of phytonematodes," in Biocontrol Agents of Phytonematodes, pp. 446-454, CABI, Wallingford, UK, 2015.

[3] A. C. Oluwaseun and N. B. Sarin, "Impacts of biogenic nanoparticle on the biological control of plant pathogens," Pollution Research, vol. 24, no. 15, pp. 13700-13709, 2017.

[4] J. W. Deacon and L. A. Berry, "Biocontrol of soil-borne plant pathogens: concepts and their application," Pesticide Science, vol. 37, no. 4, pp. 417-426, 1993.

[5] A. M. Elgorban, A. N. Al-Rahmah, S. R. Sayed, A. Hirad, A. A.-F. Mostafa, and A. H. Bahkali, "Antimicrobial activity and green synthesis of silver nanoparticles using Trichoderma viride," Biotechnology \& Biotechnological Equipment, vol. 30, no. 2, pp. 299-304, 2016.

[6] C. J. De Andrade, L. M. De Andrade, M. A. Mendes, and C. A. O. Do, "An overview on the production of microbial copper nanoparticles by bacteria, fungi and algae," Global Journal of Research in Engineering, vol. 17, no. 1, 2017.

[7] Y.-K. Jo, B. H. Kim, and G. Jung, "Antifungal activity of silver ions and nanoparticles on phytopathogenic fungi," Plant Disease, vol. 93, no. 10, pp. 1037-1043, 2009.

[8] P. Patra, S. Mitra, N. Debnath, and A. Goswami, "Biochemical-, biophysical-, and microarray-based antifungal evaluation of the buffer-mediated synthesized nano zinc oxide: an in vivo and in vitro toxicity study," Langmuir, vol. 28, no. 49, pp. 16966-16978, 2012.

[9] G. Abdelmalek and T. Salaheldin, "Silver nanoparticles as a potent fungicide for citrus phytopathogenic fungi," Journal of Nanomedicine Research, vol. 3, no. 5, Article ID 00065, 2016.

[10] P. Kanhed, S. Birla, S. Gaikwad et al., "In vitro antifungal efficacy of copper nanoparticles against selected crop pathogenic fungi," Materials Letters, vol. 115, pp. 13-17, 2014.

[11] K. Bramhanwade, S. Shende, S. Bonde, A. Gade, and M. Rai, "Fungicidal activity of $\mathrm{Cu}$ nanoparticles against Fusarium causing crop diseases," Environmental Chemistry Letters, vol. 14, no. 2, pp. 229-235, 2016.

[12] J. L. Clement and P. S. Jarrett, “Antibacterial silver," MetalBased Drugs, vol. 1, no. 5-6, pp. 467-482, 1994.

[13] H.-J. Park, S.-H. Kim, H.-J. Kim, and S.-H. Choi, "A new composition of nanosized silica-silver for control of various plant diseases," The Plant Pathology Journal, vol. 22, no. 3, pp. 295-302, 2006.

[14] S. Al-Zubaidi, A. Al-Ayafi, and H. Abdelkader, "Biosynthesis, characterization and antifungal activity of silver nanoparticles by Aspergillus niger isolate," Journal of Nanotechnology Research, vol. 1, no. 1, pp. 23-36, 2019.

[15] T. White, T. Bruns, S. Lee, and J. Taylor, "Amplification and direct sequencing of ribosomal RNA genes for phylogenetics," in PCR Protocols, I. Ma, D. H. Gelfand, J. J. Sninsky, and T. J. White), Eds., pp. 315-322, Academic Press, New York, NY, USA, 1990.
[16] S. S. Birla, S. C. Gaikwad, A. K. Gade, and M. K. Rai, "Rapid synthesis of silver nanoparticles from Fusarium oxysporum by optimizing physicocultural conditions," The Scientific World Journal, vol. 2013, Article ID 796018, 2013.

[17] S. Rajeshkumar, C. Malarkodi, K. Paulkumar, M. Vanaja, G. Gnanajobitha, and G. Annadurai, "Algae mediated green fabrication of silver nanoparticles and examination of its antifungal activity against clinical pathogens," International Journal of Metals, vol. 2014, Article ID 692643, 2014.

[18] A. Matei, S. Matei, G.-M. Matei, G. Cogălniceanu, and C. P. Cornea, "Biosynthesis of silver nanoparticles mediated by culture filtrate of lactic acid bacteria, characterization and antifungal activity," The EuroBiotech Journal, vol. 4, no. 2, pp. 97-103, 2020.

[19] R. Tripathi, R. K. Gupta, A. Shrivastav, M. P. Singh, B. Shrivastav, and P. Singh, "Trichoderma koningii assisted biogenic synthesis of silver nanoparticles and evaluation of their antibacterial activity," Advances in Natural Sciences: Nanoscience and Nanotechnology, vol. 4, no. 3, Article ID 035005, 2013.

[20] S. Zada, A. Ahmad, S. Khan et al., "Biofabrication of gold nanoparticles by Lyptolyngbya JSC-1 extract as super reducing and stabilizing agents: synthesis, characterization and antibacterial activity," Microbial Pathogenesis, vol. 114, pp. 116123, 2018.

[21] S. L. Smitha, K. M. Nissamudeen, D. Philip, and K. G. Gopchandran, "Studies on surface plasmon resonance and photoluminescence of silver nanoparticles," Spectrochimica Acta Part A: Molecular and Biomolecular Spectroscopy, vol. 71, no. 1, pp. 186-190, 2008.

[22] M. Guilger-Casagrande and R. De Lima, "Synthesis of silver nanoparticles mediated by fungi: a review," Frontiers in Bioengineering and Biotechnology, vol. 7, 2019.

[23] E. Roda, S. Barni, A. Milzani, I. Dalle-Donne, G. Colombo, and T. Coccini, "Single silver nanoparticle instillation induced early and persisting moderate cortical damage in rat kidneys," International Journal of Molecular Sciences, vol. 18, no. 10, p. 2115, 2017.

[24] C. Carlson, S. M. Hussain, A. M. Schrand et al., "Unique cellular interaction of silver nanoparticles: size-dependent generation of reactive oxygen species," The Journal of Physical Chemistry B, vol. 112, no. 43, pp. 13608-13619, 2008.

[25] S. Zada, A. Ahmad, S. Khan et al., "Biogenic synthesis of silver nanoparticles using extracts of Leptolyngbya JSC-1 that induce apoptosis in HeLa cell line and exterminate pathogenic bacteria," Artificial Cells, Nanomedicine, and Biotechnology, vol. 46, no. 3, pp. S471-S480, 2018.

[26] K. Anandalakshmi, J. Venugobal, and V. Ramasamy, "Characterization of silver nanoparticles by green synthesis method using Pedalium murex leaf extract and their antibacterial activity," Applied Nanoscience, vol. 6, no. 3, pp. 399-408, 2016.

[27] M. Kasithevar, M. Saravanan, P. Prakash et al., "Green synthesis of silver nanoparticles using Alysicarpus monilifer leaf extract and its antibacterial activity against MRSA and CoNS isolates in HIV patients," Journal of Interdisciplinary Nanomedicine, vol. 2, no. 2, pp. 131-141, 2017.

[28] S.-W. Lee, S.-H. Chang, Y.-S. Lai et al., "Effect of temperature on the growth of silver nanoparticles using plasmon-mediated method under the irradiation of green LEDs," Materials, vol. 7, no. 12, pp. 7781-7798, 2014.

[29] S. Nakamura, M. Sato, Y. Sato et al., "Synthesis and application of silver nanoparticles (Ag NPs) for the prevention of 
infection in healthcare workers," International Journal of Molecular Sciences, vol. 20, no. 15, p. 3620, 2019.

[30] S. Rajeshkumar, C. Malarkodi, G. Gnanajobitha et al., "Seaweed-mediated synthesis of gold nanoparticles using Turbinaria conoides and its characterization," Journal of Nanostructure in Chemistry, vol. 3, no. 1, p. 44, 2013.

[31] S. Devi and V. Bhimba, "Antibacterial and antifungal activity of silver nanoparticles synthesized using Hypnea muciformis," Biosciences, Biotechnology Research Asia, vol. 11, pp. 235-238, 2014.

[32] J. E. Mendes, L. Abrunhosa, J. E. Teixeira, E. R. De Camargo, C. P. De Souza, and J. D. C. Pessoa, "Antifungal activity of silver colloidal nanoparticles against phytopathogenic fungus (Phomopsis sp.) in soybean seeds," International Journal of Biological, Veterinary, Agricultural and Food Engineering, vol. 8, no. 9, pp. 928-933, 2014.

[33] P. D. Bragg and D. J. Rainnie, "The effect of silver ions on the respiratory chain of Escherichia coli," Canadian Journal of Microbiology, vol. 20, no. 6, pp. 883-889, 1974.

[34] G. McDonnell and A. D. Russell, "Antiseptics and disinfectants: activity, action, and resistance," Clinical Microbiology Reviews, vol. 12, no. 1, pp. 147-179, 1999.

[35] K.-J. Kim, W. S. Sung, B. K. Suh et al., "Antifungal activity and mode of action of silver nano-particles on Candida albicans," Biometals, vol. 22, no. 2, pp. 235-242, 2009.

[36] M. Rai, A. Yadav, and A. Gade, "Silver nanoparticles as a new generation of antimicrobials," Biotechnology Advances, vol. 27, no. 1, pp. 76-83, 2009.

[37] J. R. Morones, J. L. Elechiguerra, A. Camacho et al., "The bactericidal effect of silver nanoparticles," Nanotechnology, vol. 16, no. 10, pp. 2346-2353, 2005.

[38] V. K. Sharma, R. A. Yngard, and Y. Lin, "Silver nanoparticles: green synthesis and their antimicrobial activities," Advances in Colloid and Interface Science, vol. 145, no. 1-2, pp. 83-96, 2009. 